\title{
An assessment on the incremental value of high-resolution magnetic resonance imaging to identify culprit plaques in atherosclerotic disease of the middle cerebral artery
}

\author{
Zhongzhao Teng ${ }^{1}$ (D) - Wenjia Peng ${ }^{1,2} \cdot$ Qian Zhan ${ }^{2} \cdot$ Xuefeng Zhang ${ }^{2} \cdot$ Qi Liu $^{2}$ • \\ Shiyue Chen ${ }^{2} \cdot$ Xia Tian ${ }^{2} \cdot$ Luguang Chen ${ }^{2} \cdot$ Adam J. Brown ${ }^{3} \cdot$ Martin J. Graves $^{1} \cdot$ \\ Jonathan H. Gillard ${ }^{1}$ • Jianping Lu $^{2}$
}

Received: 20 April 2015 /Revised: 31 July 2015 / Accepted: 3 September 2015 /Published online: 16 September 2015 (C) The Author(s) 2015. This article is published with open access at Springerlink.com

\begin{abstract}
Objective Although certain morphological features depicted by high resolution, multi-contrast magnetic resonance imaging (hrMRI) have been shown to be different between culprit and non-culprit middle cerebral artery (MCA) atherosclerotic lesions, the incremental value of hrMRI to define culprit lesions over stenosis has not been assessed.

Methods Patients suspected with MCA stenosis underwent hrMRI. Lumen and outer wall were segmented to calculate stenosis, plaque burden $(\mathrm{PB})$, volume (PV), length $(\mathrm{PL})$ and minimum luminal area (MLA).

Results Data from 165 lesions (112 culprit and 53 non-culprit) in 139 individuals were included. Culprit lesions were larger and longer with a narrower lumen and increased PB compared with non-culprit lesions. More culprit lesions showed contrast enhancement. Both PB and MLA were better indicators than stenosis in differentiating lesion types (AUC were 0.649, 0.732 and 0.737 for stenosis, PB and MLA, respectively).
\end{abstract}

Zhongzhao Teng and Wenjia Peng contributed equally to this work.

Electronic supplementary material The online version of this article (doi:10.1007/s00330-015-4008-5) contains supplementary material, which is available to authorized users.

Zhongzhao Teng

zt215@cam.ac.uk

Jianping $\mathrm{Lu}$

cjr.lujianping@vip.163.com

1 Department of Radiology, University of Cambridge, Level 5, Box 218, Hills Rd., Cambridge CB2 0QQ, UK

2 Department of Radiology, Changhai Hospital, 168 Changhai Rd, Shanghai, China 200433

3 Division of Cardiovascular Medicine, University of Cambridge, Cambridge, UK
Combinations of PB, MLA and stenosis could improve positive predictive value (PPV) and specificity significantly. An optimal combination of stenosis $\geq 50 \%, P B \geq 77 \%$ and MLA $\leq 2.0 \mathrm{~mm}^{2}$ produced a $\mathrm{PPV}=85.7 \%$, negative predictive value $=54.1 \%$, sensitivity $=69.6 \%$, specificity $=75.5 \%$, and accuracy $=71.5 \%$.

Conclusions hrMRI plaque imaging provides incremental information to luminal stenosis in identifying culprit lesions.

Key points

- High resolution MRI provides incremental information in defining culprit MCA atherosclerotic lesions.

- Both plaque burden and minimum luminal area are better indicators than stenosis.

- An optimal combination includes stenosis $\geq 50 \%, P B \geq 77 \%$ and $M L A \leq 2.0 \mathrm{~mm}^{2}$.

Keywords Middle cerebral artery $\cdot$ Magnetic resonance imaging $\cdot$ Stenosis $\cdot$ Plaque burden $\cdot$ Minimum luminal area

\section{Introduction}

Intracranial atherosclerosis is a major subcategory of ischemic stroke, accounting for $\sim 10 \%$ of strokes in western societies [1], and 30-50\% of strokes in Asian populations [2, 3]. Intracranial atherosclerosis typically affects the middle cerebral artery (MCA) and the intracranial portions of the internal carotid, vertebrobasilar, posterior, and anterior cerebral arteries. Overall, about $40-70 \%$ of intracranial atherosclerotic disease is located in the MCA in Asian populations [4]. Plaque morphological and compositional features have been shown to provide incremental benefit over luminal stenosis in defining culprit lesions [5, 6] and subsequent cardiovascular ischemic events [7-9] both in the coronary and carotid circulations. 
High-resolution, multi-contrast magnetic resonance imaging (hrMRI) can delineate the structural composition within intracranial atherosclerotic plaques $[10,11]$. Studies to date have shown that symptomatic MCA plaques have larger wall area, greater remodelling ratio, higher prevalence of expansive remodelling and lower prevalence of constrictive remodelling $[12,13] . T_{1}$ - and/or $T_{2}$-hyperintense foci were also demonstrated more frequently within plaques of symptomatic patients $[14,15]$, with plaque contrast enhancement also associated with recent ischemic events [16]. Although these studies demonstrate that there is a difference between culprit and nonculprit lesions as characterized by hrMRI, the incremental value of hrMRI to identify culprit lesions, over luminal stenosis alone, is yet to be determined.

Thus, we sought to assess the incremental benefit of hrMRI imaging in identifying culprit MCA plaques as compared with luminal stenosis. Additionally, we propose an optimized combination of MCA plaque characteristics that were associated with symptomatic clinical presentation.

\section{Materials and methods}

\section{Study population}

Between January 2012 and August 2013, 154 consecutive patients with suspected intracranial atherosclerotic diseases referred from Department of Neurosurgery, Changhai Hospital, Shanghai, China, were recruited. These patients presented with symptoms and signs of MCA territory ischemia or infarction either clinically or on the basis of neurological imaging, including $\mathrm{T}_{1^{-}}, \mathrm{T}_{2^{-}}$, diffusion-weighted (DWI), $\mathrm{T}_{2}$-fluid attenuated inversion recovery (FLAIR) brain imaging and/or whole brain computerised tomography perfusion (CTP) imaging. The study protocol was approved by the local institutional review board with all patients providing written informed consent.

Patients underwent hrMRI MCA imaging with inclusion criteria being: (1) absence of significant carotid arterial stenosis $(<30 \%)$ assessed by ultrasound; (2) absence of atrial fibrillation on $24 \mathrm{hr}$ monitoring; (3) absence of ascending aortic arch atheroma on MR; and (4) $\geq 1$ atherosclerotic risk-factors, including hypertension, diabetes mellitus, hypercholesterolemia or cigarette smoking. Exclusion criteria: (1) nonatherosclerotic intracranial arterial disease, e.g. inflammatory arteritis and congenital agenesis; (2) suspected cardio-embolic stroke; (3) known coagulopathy; (4) previous MCA stenting; or (5) clinical contraindications to MRI.

One hundred and nine volunteers referred by Health Examination Centre, Changhai Hospital, were also recruited consecutively as asymptomatic controls with inclusion criteria (14) and MCA blood flow velocities $>160 \mathrm{~cm} / \mathrm{s}$ [17] assessed by transcranial Doppler or no detectable Doppler shift. There was no upper limit to MCA flow velocity and hence, volunteers with moderate/severe stenosis were not excluded. Investigators screening volunteers were blinded to their clinical status.

\section{hrMRI protocol}

Cross-sectional imaging was performed using a 3 T MR scanner (HDx, GE Healthcare, USA) and an 8-channel phased array brain coil. 3D time-of-flight (TOF) magnetic resonance angiography (MRA) was performed for positioning. Based on the use of MRA, the scan plane was angled to ensure that the cross-sectional images were perpendicular to the diseased section (Fig. 1). Black blood 2D fast spin-echo (FSE) $\mathrm{T}_{2}$-weighted $\left(T_{2}\right), T_{1}$-weighted $\left(T_{1}\right)$ and gadolinium-enhanced $T_{1}$ weighted $\left(\mathrm{CE}-\mathrm{T}_{1}\right)$ images of 12 sections were acquired consecutively. A detailed description can be found in the Supplemental Materials.

\section{MR image analyses}

Degree of luminal stenosis was computed from TOF maximum intensity projection (MIP) images following WASID criterion [18]. A plaque was identified if plaque burden $[\mathrm{PB}=($ total wall area - lumen area $) /$ total wall area $\times$ $100 \%$ ] $>40 \%$. Lesions with luminal occlusion were identified by using both TOF and $\mathrm{T}_{2}$-weighted images. Lumen and outer wall boundaries were manually segmented in each MR image in which plaque was present using CMRTools (CVIS, UK) (Fig. 2). Minimal luminal area (MLA), PB, plaque volume (PV) and plaque length (PL) were computed based on the segmentation. For those with luminal occlusion, stenosis was $100 \%$, MLA $0 \mathrm{~mm}^{2}$ and PB $100 \%$. MR signal intensity may vary within individuals due to factors such as coil positioning. Normalized signal intensity to adjacent grey matter in $\mathrm{T}_{1}, \mathrm{~T}_{2}$ and $\mathrm{CE}-\mathrm{T}_{1}$ images were therefore used for analyses (Fig. 2).

Both MLA and maximum PB along each plaque were used in subsequent analysis. A culprit plaque was defined as a lesion arising on the ipsilateral side to an ischemic stroke on neuro-imaging with accompanying clinical symptoms, whilst a non-culprit plaque was defined as either a plaque occurring in a contralateral artery of a symptomatic patient or one in asymptomatic controls. Experienced neuroradiologists and stroke physicians interpreted all clinical details and neurological imaging, but remained blinded to hrMRI data; experienced MR observers including two radiology residents and one neuroradiologist performed hrMRI analyses in agreement blinded to clinical presentation and other imaging. In total, 3 , $768 \mathrm{~T}_{1}, \mathrm{~T}_{2}$ and CE-T $\mathrm{T}_{1}$ MR slices were reviewed and 2,379 of them were identified as containing MCA plaque, with 1,581 of these $(66.5 \%)$ arising from culprit lesions. 
Fig. 1 MRI images showing an MCA atherosclerotic plaque in a symptomatic patient. A mixture of fresh and chronic infarction involving the right periventricular and frontal subcortical regions was seen in DWI (red dashed curve); the plaque is shown by an arrow in the TOF image, and a cross section at MLA is shown in $\mathrm{T}_{2}, \mathrm{~T}_{1}$ and CE- $\mathrm{T}_{1}$ images (white arrows: plaque and red asterisks: lumen)

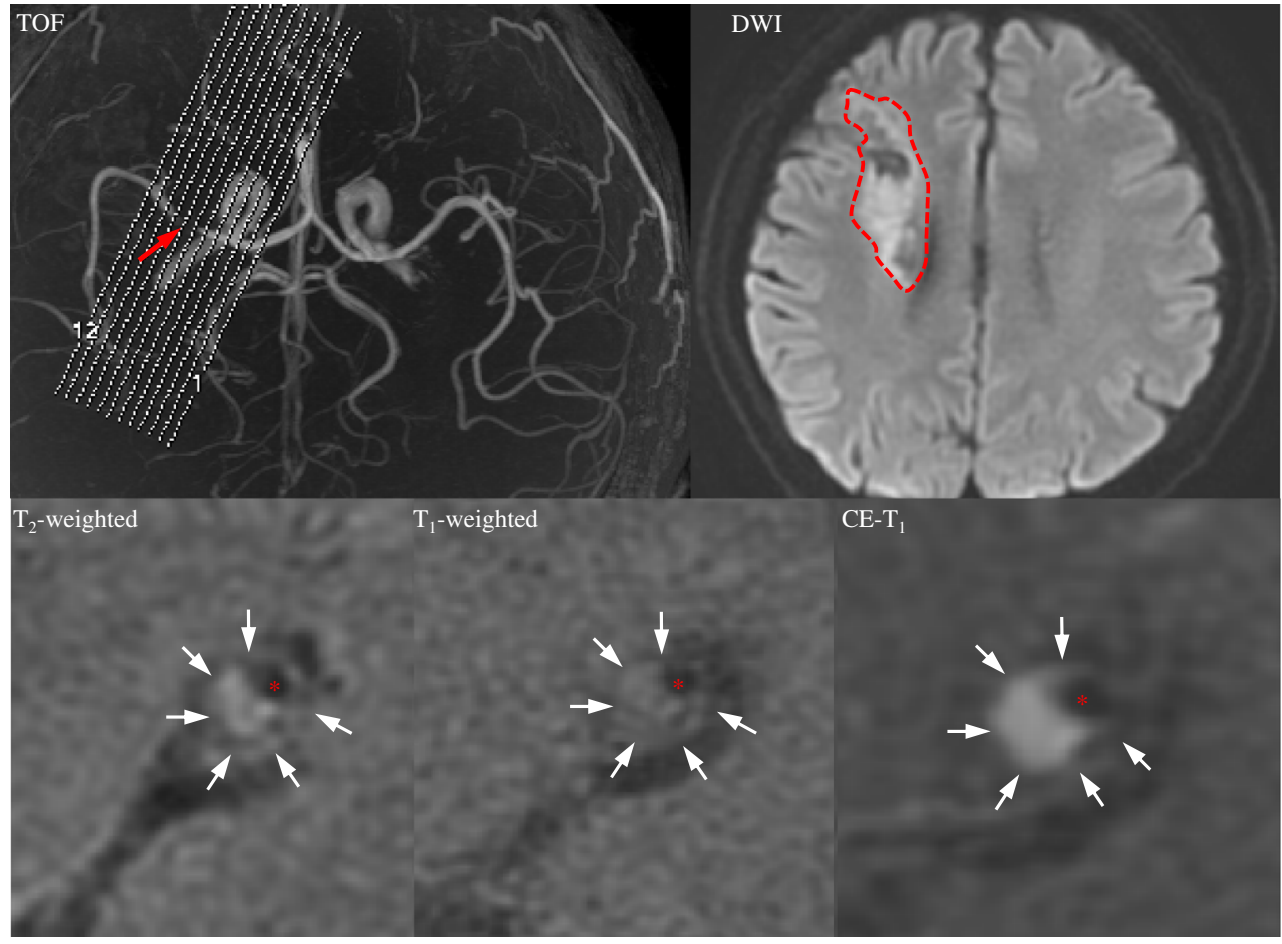

\section{Statistical analyses and optimization procedures}

Data was assessed for normality by using the Shapiro-Wilk test. Continuous data were presented as either mean (SD) or median [Q1-Q3], while categorical data were expressed as counts or percentages. Continuous data were compared using either a Mann-Whitney or students $t$ test, as appropriate. Categorical variables were analysed using Fisher's exact test. As each plaque provided multiple MR images, a linear mixed effect model was employed to compare the normalized signal intensity in $T_{1}, T_{2}$ and CE- $T_{1}$ images between groups. The receiver operating characteristic curve (ROC) analysis and comparisons were performed using MedCalc 13.3.0.0
(MedCalc Software, Belgium). To determine the effect of lesion characteristics on plaque status, univariate and multivariate models were constructed. Variables reaching significance on univariate analysis were entered into the multivariate model and are presented as odds rations with $95 \%$ confidence intervals. Except for ROC analysis, all other statistical analyses were two-tailed and performed in R 2.10.1 (The R Foundation for Statistical Computing). Statistical significance was assumed if $p<0.05$.

To define optimized cutoff values for specific plaque features to identify a culprit lesion, a procedure was designed in Matlab R2014a (The MathWorks Inc., USA) to find the optimal cutoffs by maximizing the objective

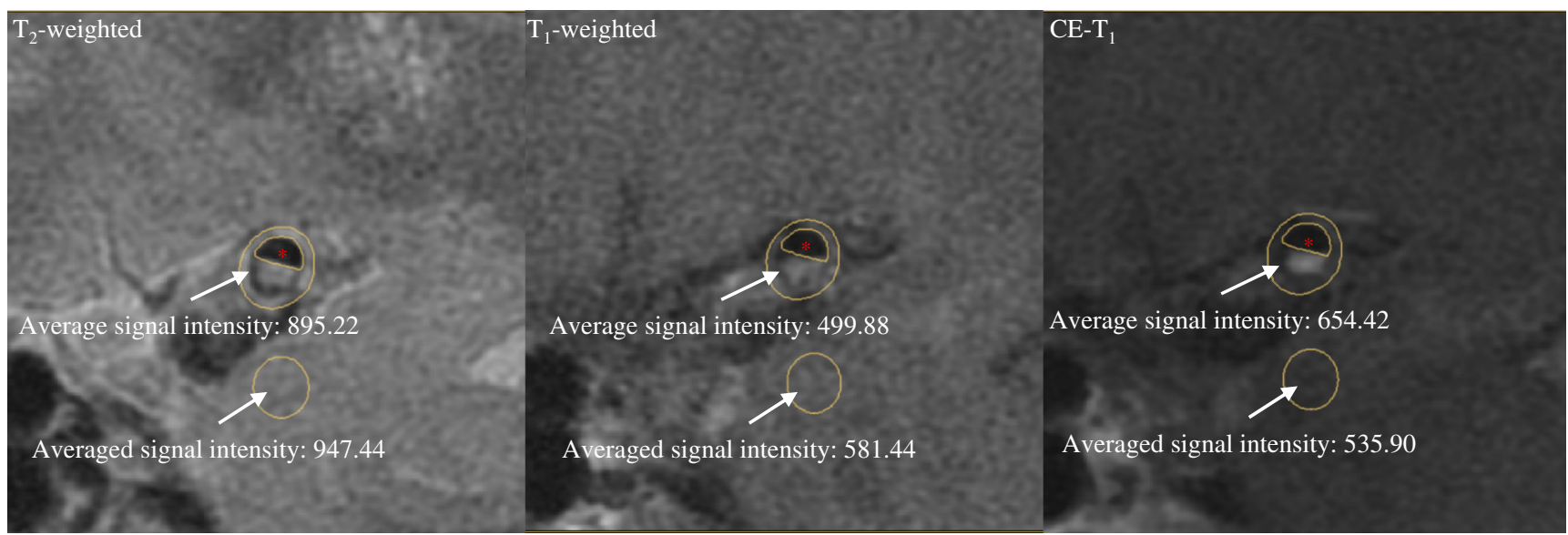

Fig. 2 Calculation of normalized signal intensity. The averaged signal intensity over the pixels enclosed between the lumen and outer wall was normalized by the value obtained from the adjacent grey matter with an area of 10-12 $\mathrm{mm}^{2}$ (red asterisks: lumen) 
function, $\psi=\mathrm{PPV} \times \mathrm{NPV} \times$ Sensitivity $\times$ Specificity $\times$ Accuracy (PPV and NPV stand for positive and negative predictive values, respectively), with the assumption that each parameter was equally important. In this produce, an unconstrained nonlinear optimization function, fminsearch(), was used. It uses a simplex search method without involving numerical or analytic gradients.

\section{Results}

\section{Patient demographics}

The baseline patient demographics are presented in Table 1. Both total cholesterol and low-density lipoprotein in the symptomatic group were higher than those in the asymptomatic group; however, no other significant differences were observed.

MCA atherosclerosis was observed in 112 patients $(72.7 \%)$ in the symptomatic cohort, 26 of which had bilateral lesions (Fig. 3). MCA atherosclerosis was found in 27 volunteers $(24.8 \%)$ with none having bilateral lesions. Of all 165 lesions, 152 (92.1\%) were located in M1 section and $13(7.9 \%)$ in proximal M2 section. In the symptomatic cohort with atherosclerosis, six $(5.4 \%)$ patients suffered from transient ischemic attack (TIA) and 106 (94.6\%) from a stroke. Eighty-two (73.2\%) patients showed DWIpositive infarction, with the majority $(n=56)$ having predominantly subcortical infarcts, nine pial territory infarcts with or without insular infarcts, ten cortical border-zone infarcts, and seven isolated small subcortical infarcts. Seventy-two patients had either acute or acute/chronic infarctions, while only ten had chronic infarctions. Five TIA patients had normal DWI and apart from these five patients, 17 with DWI-negative showed mild ischemic perfusion changes on ipsilateral MCA territory, i.e., increased time to peak, characterized by CTP. In the asymptomatic cohort, only one had evidence of chronic infarction.

\section{Luminal stenosis and plaque type}

In this study, stenosis category was defined according to TOF-stenosis as, no stenosis: $<29 \%$; mild stenosis: 30-49 \%; moderate stenosis: 50-69 \%; severe stenosis: 70-99\% and occlusion. As presented in Table 2, culprit lesions stenosis was significantly greater than non-culprit lesion stenosis $(p=0.002) .92$ culprit lesions $(82.1 \%)$ had moderate or greater stenosis, whereas 32 non-culprit lesions $(60.4 \%)$ fell within this category $(p=0.005)$. The comparisons of stenosis and the number of lesions within each stenosis category between symptomatic patients with both culprit and contralateral non-culprit lesions are presented in Supplemental Materials. No significant differences were found between non-culprit lesions in symptomatic and asymptomatic patients.

ROC analysis revealed the area under the curve (AUC) for luminal stenosis alone to define culprit lesions to be 0.649 (95\% Confidence Interval (CI) [0.571, 0.722]) (Fig. 4A). The optimized stenosis cutoff for differentiating culprit and non-culprit lesions was $54 \%$, resulting in a sensitivity and specificity of $70.5 \%$ and $56.6 \%$, respectively.

\section{hrMRI and plaque type}

As listed in Table 2, PB and PV of culprit lesions were both significantly larger than those of non-culprit lesions $(p<0.001$ and $p=0.02$, respectively), with lower plaque MLA $(p<0.001)$ and increased PL $(p=0.002)$. In our study, seven $(6.3 \%)$ culprit and one (3.7\%) non-culprit lesions had MR slices with normalized $\mathrm{T}_{1}$ signal intensity $>1.0$; and 27 (24.1\%) culprit

Table 1 Patient demographics

\begin{tabular}{lccc}
\hline & $\begin{array}{l}\text { Symptomatic } \\
(\mathrm{n}=112)\end{array}$ & $\begin{array}{l}\text { Asymptomatic } \\
(\mathrm{n}=27)\end{array}$ & $p$ value \\
\hline Male, $\mathrm{n}(\%)$ & $75(67.0)$ & $15(55.6)$ & 0.374 \\
Age, $($ Mean $\pm \mathrm{SD})$ & $56.8 \pm 10.2$ & $58.6 \pm 11.1$ & 0.448 \\
Smoke, $\mathrm{n}(\%)$ & $36(32.1)$ & $5(18.5)$ & 0.239 \\
Hypertension, $\mathrm{n}(\%)$ & $81(68.1)$ & $18(66.7)$ & 0.730 \\
Diabetes mellitus, $\mathrm{n}(\%)$ & $39(34.8)$ & $9(33.3)$ & 0.884 \\
Ischemic heart disease, $\mathrm{n}(\%)$ & $8(7.1)$ & $5(18.5)$ & 0.131 \\
Pre-admission aspirin, $\mathrm{n}(\%)$ & $47(42.0)$ & $10(37.0)$ & 0.803 \\
Pre-admission statin, $\mathrm{n}(\%)$ & $37(36.3)$ & $8(29.6)$ & 0.912 \\
Total cholesterol, (mmol/l, mean $\pm \mathrm{SD})$ & $4.44 \pm 0.94$ & $3.74 \pm 1.00$ & 0.005 \\
Triglyceride, $(\mathrm{mmol} / \mathrm{l}, \mathrm{mean} \pm \mathrm{SD})$ & $1.67 \pm 1.34$ & $1.60 \pm 0.52$ & 0.175 \\
High-density lipoprotein, (mmol/1, mean $\pm \mathrm{SD})$ & $1.10 \pm 0.33$ & $1.08 \pm 0.22$ & 0.826 \\
Low-density lipoprotein, $(\mathrm{mmol} / \mathrm{l}, \mathrm{mean} \pm \mathrm{SD})$ & $2.54 \pm 0.65$ & $1.89 \pm 0.72$ & 0.003 \\
Days between hrMRi imaging and admission (mean $\pm \mathrm{SD})$ & $3.3 \pm 2.5$ & - & \\
\hline
\end{tabular}




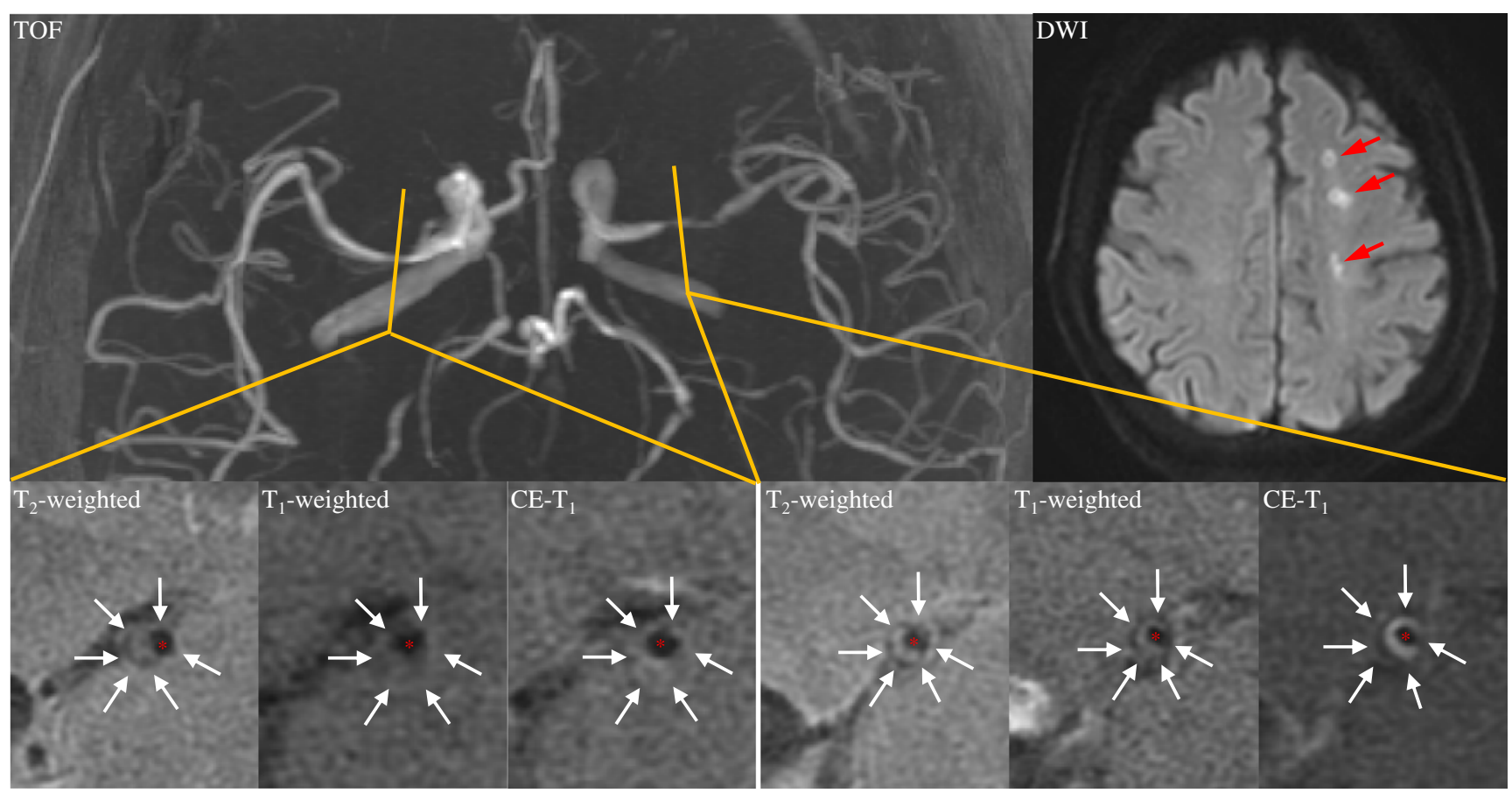

Fig. 3 A symptomatic patient with bilateral atherosclerotic lesions. The culprit lesion was located on the left side of MCA as confirmed by acute ipsilateral multiple subcortical infarcts on DWI (marked by red arrows)

and $12(22.6 \%)$ non-culprit lesions had MR slices with normalized $\mathrm{T}_{2}$ signal intensity $>1.0$ (Table 2 ). Among those MR slices with higher $\mathrm{T}_{2}$ signal intensity, the majority $(90.6 \%$ for culprit and $100 \%$ for non-culprit) had normalized $\mathrm{T}_{1}$ signal intensity $<1.0$, suggesting fibrous tissue at these locations. However, no significant difference was observed in terms of either normalized $\mathrm{T}_{1}(p=0.445)$ or $\mathrm{T}_{2}(p=0.858)$ signal intensity. More culprit lesions showed enhancement in CE- $\mathrm{T}_{1}$ images $(48.2 \%$ vs. $17.0 \%$; $p=0.001)$.

\section{The incremental value of hrMRI}

These data suggest that hrMRI-depicted MLA, PB, PV, PL and contrast enhancement are all potentially capable of differentiating culprit from non-culprit lesions. To ascertain whether these features significantly improved the capability of imaging to identify clinical presentation, serial ROC analyses were performed (Fig. 4). The AUC of PB $(0.73295 \% \mathrm{CI}[0.658$, 0.798]; Optimal criterion $\geq 90 \%)$ and MLA $(0.73795 \%$ CI

Table 2 Comparisons of plaque characteristics between culprit and non-culprit lesions

\begin{tabular}{|c|c|c|c|c|}
\hline & \multicolumn{2}{|c|}{ Symptomatic patient $(\mathrm{n}=112)$} & \multirow[t]{2}{*}{ Asymptomatic patient $(\mathrm{n}=27)$} & \multirow[t]{2}{*}{$p$ value } \\
\hline & Culprit $(n=112)$ & Contralateral non-culprit $(n=26)$ & & \\
\hline TOF-stenosis, median [IQR] (\%) & $67.3[54.1,80.5]$ & $48.8[32.4,72.3]$ & $55.3[47.0,72.6]$ & 0.002 \\
\hline No stenosis $(<29 \%), \mathrm{n}(\%)$ & $2(1.8)$ & $5(19.2)$ & $3(11.1)$ & 0.003 \\
\hline Mild (30-49 \%), n (\%) & $18(16.1)$ & $8(30.8)$ & $5(18.5)$ & 0.278 \\
\hline Moderate (50-69\%), n (\%) & $40(35.7)$ & $5(19.2)$ & $12(44.4)$ & 0.777 \\
\hline Severe $(70-99 \%), \mathrm{n}(\%)$ & $25(22.3)$ & $5(19.2)$ & $4(14.8)$ & 0.558 \\
\hline Occlusion, $\mathrm{n}(\%)$ & $27(24.1)$ & $3(11.6)$ & $3(11.1)$ & 0.088 \\
\hline Plaque burden $(\%)$ & $91[84,96]$ & $75[66,88]$ & $83[74,89]$ & $<0.0001$ \\
\hline Minimum luminal area $\left(\mathrm{mm}^{2}\right)$ & $0.95[0.43,1.94]$ & $3.12[1.66,5.06]$ & $2.22[1.08,3.15]$ & $<0.0001$ \\
\hline Plaque volume $\left(\mathrm{mm}^{3}\right)$ & $45.27[23.93,61.80]$ & $27.82[19.20,44.29]$ & $29.35[21.14,43.20]$ & 0.020 \\
\hline Plaque length (mm) & $8[6,12]$ & $6[4,9.5]$ & $8[4,8]$ & 0.002 \\
\hline Normalized $\mathrm{T}_{1}$ signal intensity $>1.0, \mathrm{n}(\%)$ & $7(6.3)$ & $0(0)$ & $1(3.7)$ & 0.439 \\
\hline Normalized $\mathrm{T}_{2}$ signal intensity $>1.0, \mathrm{n}(\%)$ & $27(24.1)$ & $4(15.4)$ & $8(29.6)$ & 0.953 \\
\hline Normalized CE- $\mathrm{T}_{1}$ signal intensity $>1.0, \mathrm{n}(\%)$ & $54(48.2)$ & $2(7.7)$ & $7(25.9)$ & 0.0002 \\
\hline
\end{tabular}



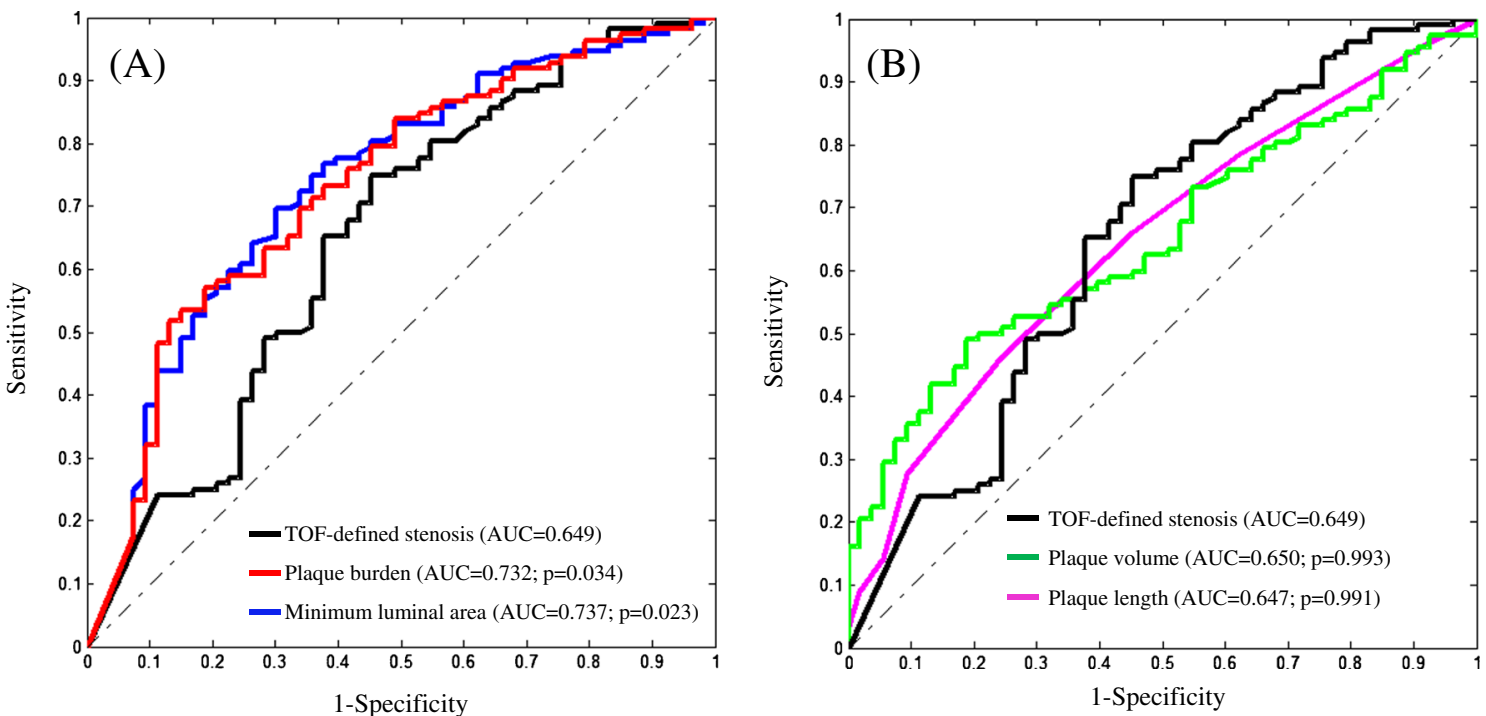

Fig. 4 Comparisons of ROC curves using stenosis, PB, MLA, PV and PL to determine a culprit plaque responsible for clinical presentation. (A: AUCs of PB and MLA are significantly higher than the one of stenosis; and B: AUCs of stenosis, PV and PL are comparable)

[0.663, 0.803]; Optimal criterion $\leq 1.6 \mathrm{~mm}^{2}$ ) were significantly higher than the AUC determined by luminal stenosis, with an incremental increase of approximately $15 \%$ (Fig. 4A). However, stepwise analyzes of classification functions, as shown in Table 3, suggest that combinations of PB, MLA and enhancement in each different stenosis category did not improve the overall diagnostic accuracy significantly [Accuracy $=($ True positive + True negative $) /$ Total lesions]. In each stenosis category, by increasing PB, decreasing MLA or adding enhancement, PPV and specificity increase, whereas NPV and sensitivity decrease. As shown in Fig. 4B, the AUCs of PV and PL were both comparable with the one of luminal stenosis $(p>0.05)$. Overall hrMRI provides an incremental value to stenosis, adding one of the features of $\mathrm{PB} \geq 90 \%$, MLA $\leq 1.6 \mathrm{~mm}^{2}$ and enhancement will increase PPV and specificity up to over $80 \%$ in all stenosis catalogues.

\section{The optimal combination}

Univariate regression analysis suggested that stenosis, PB, MLA, PV, PL and enhancement were predictors in differentiating culprit from non-culprit lesions (Table 4). However, multivariate regression analysis indicated that none of these variables alone was independently predictive $(p>0.05)$, suggesting that a combination approach may therefore be required. An optimization procedure was designed to define the optimal cutoff for combinations of plaque features to identify culprit lesions. We found that stenosis $\geq 50 \%, P B \geq 77 \%$, and $\mathrm{MLA} \leq 2.0 \mathrm{~mm}^{2}$ was the optimal combination, producing $\mathrm{PPV}=85.7 \%, \mathrm{NPV}=54.1 \%$, sensitivity $=69.6 \%$, specificity $=75.5 \%$, and diagnostic accuracy $=71.5 \%$. As shown in Table 4, multivariate regression analysis using this combined variable along with other plaque characteristics (PV, PL and enhancement) demonstrated that it was independently associated with culprit plaques (odds ratio: 3.99 [1.74, 9.13]; $p=0.001$ ).

\section{Discussion}

Our study demonstrates that MCA culprit lesions exhibit a greater PB and PV, reduced MLA and greater PL than nonculprit controls, with more culprit lesions demonstrate enhancement on CE- $\mathrm{T}_{1}$ imaging. Both MLA and PB have a higher discriminatory power with which to identify plaque responsible for a symptomatic presentation, as compared with luminal stenosis alone. Finally, our analysis shows that combinations of MLA, PB and stenosis improves the PPV and specificity for MR-imaging to identify culprit plaques. Based on these data, the optimal combination would be stenosis $\geq$ $50 \%, \mathrm{~PB} \geq 77 \%$, and MLA $\leq 2.0 \mathrm{~mm}^{2}$.

The limitation of luminal stenosis to assess disease severity has been widely demonstrated. Myocardial infarctions are known to occur in mild or moderate lesions on angiography [19], while patients with mild to moderate carotid stenosis constitute the majority of individuals suffering from clinical events [20]. These pathological observations have also been confirmed in MCA, as lesions with moderate stenosis are frequently responsible for dependent territorial stroke [21]. In addition, as shown in Fig. 2, the lumen shape may be irregular in the diseased region and the MIP TOF-based stenosis measurement would therefore be projection direction-dependent. As shown in Fig. 5, when data from all lesions were pooled, a moderate correlation between MLA and stenosis was observed (Fig. $5 \mathrm{~A} ; \mathrm{R}^{2}=0.41$ ). However, when data from lesions with stenosis $\leq 70 \%$ were analysed, a poor correlation was 
Table 3 Predictive values, sensitivities and specificities of individual characteristics depicted by hrMRI

\begin{tabular}{llllll}
\hline & PPV (\%) & NPV (\%) & Sensitivity (\%) & Specificity (\%) & Diagnostic accuracy (\%) \\
\hline Stenosis $\geq 30 \%$ & 71.0 & 80.0 & 98.2 & 15.1 & 71.5 \\
$+\mathrm{PB} \geq 60 \%$ & 71.9 & 83.3 & 98.2 & 18.9 & 72.7 \\
$+\mathrm{PB} \geq 75 \%$ & 76.0 & 61.1 & 87.5 & 41.5 & 72.7 \\
$+\mathrm{PB} \geq 90 \%$ & 85.9 & 45.7 & 54.5 & 81.1 & 63.0 \\
+ MLA $\leq 1.0 \mathrm{~mm}^{2}$ & 86.8 & 45.4 & 52.7 & 83.0 & 62.4 \\
+ MLA $\leq 2.0 \mathrm{~mm}^{2}$ & 81.6 & 54.8 & 75.0 & 64.2 & 71.5 \\
$+\mathrm{MLA} \leq 4.0 \mathrm{~mm}^{2}$ & 74.3 & 68.0 & 92.9 & 32.1 & 73.3 \\
+ Enhancement & 85.5 & 42.7 & 47.3 & 83.0 & 58.8 \\
Stenosis $\geq 50 \%$ & 74.2 & 51.2 & 82.1 & 39.6 & 68.5 \\
$+\mathrm{PB} \geq 60 \%$ & 74.8 & 52.4 & 82.1 & 41.5 & 69.1 \\
$+\mathrm{PB} \geq 75 \%$ & 78.2 & 52.7 & 76.8 & 54.7 & 69.7 \\
$+\mathrm{PB} \geq 90 \%$ & 86.7 & 45.4 & 52.7 & 83.0 & 62.4 \\
$+\mathrm{MLA} \leq 1.0 \mathrm{~mm}^{2}$ & 86.8 & 45.4 & 52.7 & 83.0 & 62.4 \\
$+\mathrm{MLA} \leq 2.0 \mathrm{~mm}^{2}$ & 83.9 & 52.8 & 69.6 & 71.7 & 70.3 \\
+ +MLA $\leq 4.0 \mathrm{~mm}^{2}$ & 75.7 & 50.0 & 77.7 & 47.2 & 67.9 \\
+ Enhancement & 86.0 & 41.7 & 43.8 & 84.9 & 57.0 \\
Stenosis $\geq 70 \%$ & 77.6 & 38.8 & 46.4 & 71.7 & 54.5 \\
+ PB $\geq 60 \%$ & 77.6 & 38.8 & 46.4 & 71.7 & 54.5 \\
+ PB $\geq 75 \%$ & 78.8 & 39.4 & 46.4 & 73.6 & 55.2 \\
+ PB $\geq 90 \%$ & 85.7 & 39.7 & 37.5 & 86.8 & 53.3 \\
+ MLA $\leq 1.0 \mathrm{~mm}^{2}$ & 85.7 & 39.7 & 37.5 & 86.8 & 53.3 \\
+ MLA $\leq 2.0 \mathrm{~mm}^{2}$ & 82.3 & 40.8 & 45.5 & 79.2 & 56.4 \\
$+\mathrm{MLA} \leq 4.0 \mathrm{~mm}^{2}$ & 77.6 & 38.8 & 46.4 & 71.7 & 54.5 \\
+ Enhancement & 81.6 & 36.2 & 27.7 & 86.8 & 46.7 \\
\hline
\end{tabular}

observed (Fig. 5B; $\mathrm{R}^{2}=0.15$ ). In this study, $53.6 \%$ culprit and $71.7 \%$ non-culprit lesions are with stenosis $\leq 70 \%$. These may explain partially why MLA is more powerful than stenosis in differentiating clinical presentations (Fig. 4A).

Compared with asymptomatic lesions, previous studies have shown that symptomatic MCA plaques have a larger wall area, greater remodelling ratio, higher prevalence of expansive remodelling and lower prevalence of constrictive remodelling $[12,15]$. Our study ascertained quantitatively that PB and MLA depicted by hrMRI provide adding value in differentiating culprit from non-culprit lesions in MCA. Similar results have been obtained in the coronary circulation where MLA $\leq 4 \mathrm{~mm}^{2}$ was found to be associated with acute syndrome [22] and MLA $\leq 4 \mathrm{~mm}^{2}$ and $\mathrm{PB} \geq 70 \%$ are predictive for future cardiovascular events [7, 8]. As luminal and out wall contours can be identified accurately by hrMRI with excellent intra-observer and inter-observer reproducibility [23], PB and MLA should be considered for future prospective trials seeking optimal management strategies for MCA atherosclerotic diseases.

Although this study has demonstrated the value of $\mathrm{PB}$ and MLA in defining culprit MCA lesions, the improvement is insufficient for application in clinical practice. Diagnostic accuracy may be improved through integration of other hrMRI

Table 4 Univariate and multivariate regression analyses showing the odds ratio (OR) for plaque characteristics (CI: confidence interval)

\begin{tabular}{|c|c|c|c|c|c|c|c|}
\hline & \multicolumn{3}{|c|}{ Univariate regression analysis } & & \multicolumn{3}{|c|}{ Multivariate regression analysis } \\
\hline & OR & $95 \% \mathrm{CI}$ & $p$ value & & OR & $95 \% \mathrm{CI}$ & $p$ value \\
\hline Stenosis & 1.02 & $1.01-1.04$ & 0.002 & Combination: & 3.99 & $1.74-9.13$ & 0.001 \\
\hline Plaque burden & 1.07 & $1.04-1.11$ & $<0.0001$ & Stenosis $\geq 50 \%+$ Plaque burden $\geq 77 \%+$ & & & \\
\hline Minimum luminal area & 0.64 & $0.52-0.78$ & $<0.0001$ & Minimum luminal area $\leq 2.0 \mathrm{~mm}^{2}$ & & & \\
\hline Plaque volume & 1.03 & $1.01-1.05$ & 0.001 & Plaque volume & 1.03 & $0.99-1.06$ & 0.14 \\
\hline Plaque length & 1.35 & $1.11-1.64$ & 0.002 & Plaque length & 0.95 & $0.65-1.37$ & 0.77 \\
\hline Enhancement & 4.55 & $2.03-10.20$ & $<0.0001$ & Enhancement & 2.27 & $0.90-5.71$ & 0.08 \\
\hline
\end{tabular}


(A)

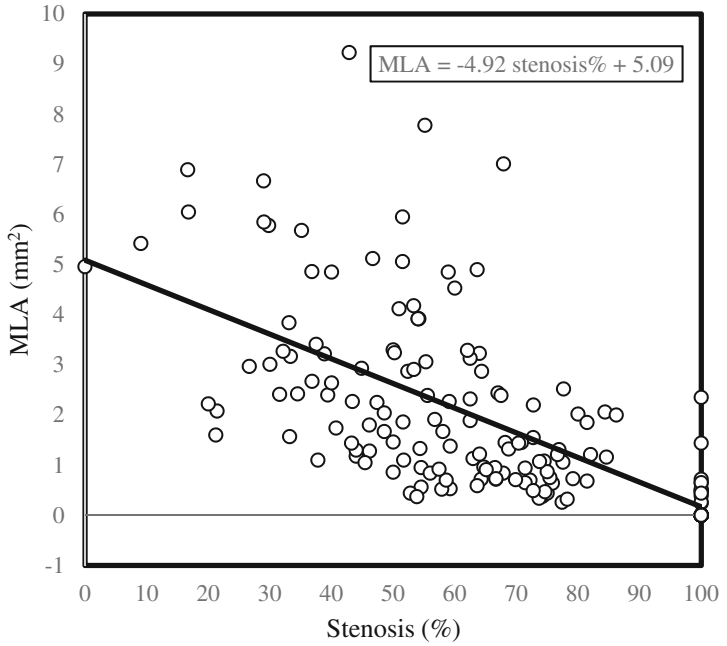

Fig. 5 The correlation between minimum luminal area and stenosis. ( $A$ : There is a good correlation between MLA and stenosis when data from all lesions are pooled $\left(\mathrm{R}^{2}=0.41 ; p<0.0001\right) ; B$ : A poor correlation exists if

features, including plaque composition. The importance of defining plaque compositional features has been advocated in carotid atherosclerosis [5], with higher-risk plaques being characterized by the presence of intraplaque haemorrhage (IPH), a large lipid-rich core and thin fibrous cap. These features can be quantified accurately by in vivo hrMRI [5], and can predict future cerebrovascular ischemic events [24]. Encouraged by these observations, hrMRI has been introduced to delineate the structural composition within intracranial atherosclerotic plaques [10, 11, 14]. However, intracranial hrMRI poses a much greater challenge given the small size and deep location of the target vessel. Despite these challenges, we observed low prevalence of high signal intensity in $T_{1}$ images in culprit lesions, consistent with previous studies [15], implying a lack of IPH/thrombus in MCA lesions. Our study also confirmed that more symptomatic lesions demonstrated contrast enhancement [16]. However, as shown in Table 3, adding enhancement did not improve the diagnostic accuracy, possibly because it led to relatively lower sensitivity and smaller NPV. Further analysis indicated that AUC for enhancement was 0.656 , which was comparable with the one at of stenosis $(p>0.05)$. However, it has to be pointed out that in this study, the signal intensity in each MR sequence was averaged over the plaque cross section, such that focal hyperintensity could have been underestimated (Fig. 2).

The incremental value of anatomic features, including IPH, thrombus and fibrous cap defect, depicted by hrMRI in differentiating patient clinical presentations and predicting subsequent ischaemic events could be quantified with further advancement in MR sequences development. Additionally, as patient clinical presentation occurs following MCA plaque rupture, calculation of plaque structural stress may improve the diagnostic power of imaging to identify culprit lesions.
(B)

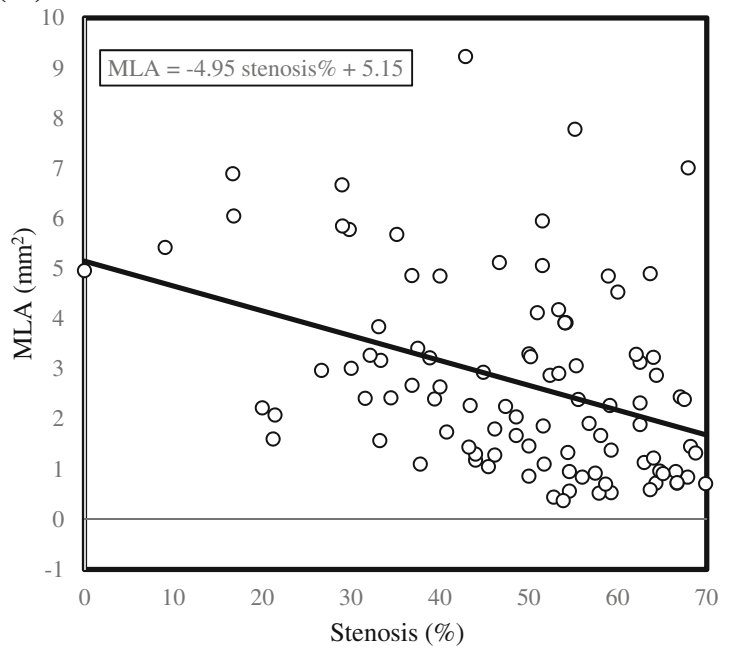

data from lesions with severe stenosis and occlusion are excluded $\left(\mathrm{R}^{2}=\right.$ $0.15 ; p<0.0001)$

Early results in the carotid and coronary circulation have been encouraging $[25,26]$, and it remains to be seen whether this technique can be clinically applied.

There are some limitations to this study: (1) this is a retrospective study and a comprehensive prospective study with follow-up is needed to ascertain the complementary value of hrMRI; (2) partial volume effect may be induced by the 2-mm slice thickness. Novel MR-based imaging techniques, such as 3D FSE and reduced field of view, are therefore required to improve the visualisation of fine structures; (3) due to the limited resolution, plaque characteristics were assessed according to features within the area enclosed by lumen and outer wall boundaries. The healthy arterial wall might have been included. Moreover this averaging approach also prevented a component-specific analysis. The potential of atherosclerotic components in differentiating plaque types was therefore not assessed; (4) in this study, the identification of culprit lesions was based on the best experience of two neuroradiologists and one stroke physician. However, lesions might exist in other locations, which were responsible for the symptom but without causing significant luminal stenosis; (5) in this study, ten culprit lesions from patients with chronic infarcts were included. As plaque characteristics may change over time [27], plaque characteristics of these ten lesions might be different from those in acute phase. However, further analysis indicated that stenosis, PB, MLA, PV, PL and enhancement (Table II in the Supplemental Materials) of these ten lesions were similar with the entire culprit cohort, and the conclusion would not change if data from these ten lesions were excluded; and (6) there was a high prevalence of moderate/severe stenosis $(59.3 \%)$ in the asymptomatic cohort and this might have affected plaque characteristics. 
In conclusion, we show that hrMRI-defined MLA and PB are both better at discriminating clinical presentations in patients with MCA atherosclerosis than luminal stenosis. Combinations of MLA, PB and enhancement improve the PPV and specificity significantly.

Acknowledgments The scientific guarantors of this publication are Dr Teng and Professor Lu. The authors of this manuscript declare no relationships with any companies, whose products or services may be related to the subject matter of the article. This study has received funding by the 12th Five Year Plan medical key project PLA, China (BWS12J026), the Emerging Frontier Technology Joint Research Program of Shanghai Municipal Hospital, China (SHDC12013110), the National Nature Science Foundation, China (31470910) and British Heart Foundation (PG/11/74/ 29100). Dr Peng is supported by China Scholarship Council (201306580006), Beijing, China. One of the authors has significant statistical expertise. Institutional Review Board approval was obtained. Written informed consent was obtained from all subjects (patients) in this study. Methodology: prospective, observational, performed at one institution.

Open Access This article is distributed under the terms of the Creative Commons Attribution-NonCommercial 4.0 International License (http:// creativecommons.org/licenses/by-nc/4.0/), which permits any noncommercial use, distribution, and reproduction in any medium, provided you give appropriate credit to the original author(s) and the source, provide a link to the Creative Commons license, and indicate if changes were made.

\section{References}

1. White $H$, Boden-Albala B, Wang $C$ et al (2005) Ischemic stroke subtype incidence among whites, blacks, and hispanics: the northern Manhattan study. Circulation 111:1327-1331

2. Wong KS, Huang YN, Gao S, Lam WW, Chan YL, Kay R (1998) Intracranial stenosis in chinese patients with acute stroke. Neurology 50:812-813

3. De Silva DA, Woon FP, Lee MP, Chen CP, Chang HM, Wong MC (2007) South Asian patients with ischemic stroke: intracranial large arteries are the predominant site of disease. Stroke 38:2592-2594

4. Kim JS, Caplan LR, Wang LK (2008) Intracranial atherosclerosis. Wiley, West Sussex

5. Underhill HR, Hatsukami TS, Fayad ZA, Fuster V, Yuan C (2010) Mri of carotid atherosclerosis: clinical implications and future directions. Nat Rev Cardiol 7:165-173

6. Garcia-Garcia HM, Costa MA, Serruys PW (2010) Imaging of coronary atherosclerosis: intravascular ultrasound. Eur Heart J 31: 2456-2469

7. Stone GW, Maehara A, Lansky AJ et al (2011) A prospective natural-history study of coronary atherosclerosis. N Engl J Med 364:226-235

8. Calvert PA, Obaid DR, O'Sullivan M et al (2011) Association between ivus findings and adverse outcomes in patients with coronary artery disease: the viva (vh-ivus in vulnerable atherosclerosis) study. JACC Cardiovasc Imaging 4:894-901

9. Saam T, Hetterich H, Hoffmann V et al (2013) Meta-analysis and systematic review of the predictive value of carotid plaque hemorrhage on cerebrovascular events by magnetic resonance imaging. J Am Coll Cardiol 62:1081-1091
10. Klein IF, Lavallee PC, Touboul PJ, Schouman-Claeys E, Amarenco $P$ (2006) In vivo middle cerebral artery plaque imaging by highresolution mri. Neurology 67:327-329

11. Turan TN, Bonilha L, Morgan PS, Adams RJ, Chimowitz MI (2011) Intraplaque hemorrhage in symptomatic intracranial atherosclerotic disease. J Neuroimaging 21:e159-e161

12. $\mathrm{Xu}$ WH, Li ML, Gao S et al (2010) In vivo high-resolution $\mathrm{mr}$ imaging of symptomatic and asymptomatic middle cerebral artery atherosclerotic stenosis. Atherosclerosis 212:507-511

13. Chung GH, Kwak HS, Hwang SB, Jin GY (2012) High resolution $\mathrm{mr}$ imaging in patients with symptomatic middle cerebral artery stenosis. Eur J Radiol 81:4069-4074

14. Ryu CW, Jahng GH, Kim EJ, Choi WS, Yang DM (2009) High resolution wall and lumen mri of the middle cerebral arteries at 3 tesla. Cerebrovasc Dis 27:433-442

15. Xu WH, Li ML, Gao S et al (2012) Middle cerebral artery intraplaque hemorrhage: prevalence and clinical relevance. Ann Neurol 71:195-198

16. Qiao Y, Zeiler SR, Mirbagheri S et al (2014) Intracranial plaque enhancement in patients with cerebrovascular events on highspatial-resolution mr images. Radiology 271:534-542

17. Gao S, Lam WW, Chan YL, Liu JY, Wong KS (2002) Optimal values of flow velocity on transcranial doppler in grading middle cerebral artery stenosis in comparison with magnetic resonance angiography. J Neuroimaging 12:213-218

18. Chimowitz MI, Lynn MJ, Howlett-Smith $\mathrm{H}$ et al (2005) Comparison of warfarin and aspirin for symptomatic intracranial arterial stenosis. N Engl J Med 352:1305-1316

19. Little WC, Constantinescu M, Applegate RJ et al (1988) Can coronary angiography predict the site of a subsequent myocardial infarction in patients with mild-to-moderate coronary artery disease? Circulation 78:1157-1166

20. Group ECSTC (1998) Randomised trial of endarterectomy for recently symptomatic carotid stenosis: final results of the mrc European carotid surgery trial (ecst). Lancet 351:1379-1387

21. Klein IF, Labreuche J, Lavallee PC et al (2010) Is moderate atherosclerotic stenosis in the middle cerebral artery a cause of or a coincidental finding in ischemic stroke? Cerebrovasc Dis 29:140-145

22. Murray SW, Stables RH, Garcia-Garcia HM et al (2014) Construction and validation of a plaque discrimination score from the anatomical and histological differences in coronary atherosclerosis: the liverpool ivus-v-heart (intra vascular ultrasound-virtualhistology evaluation of atherosclerosis requiring treatment) study. EuroIntervention 10:815-823

23. Yang WQ, Huang B, Liu XT, Liu HJ, Li PJ, Zhu WZ (2014) Reproducibility of high-resolution mri for the middle cerebral artery plaque at 3t. Eur J Radiol 83:e49-e55

24. Takaya N, Yuan C, Chu B et al (2006) Association between carotid plaque characteristics and subsequent ischemic cerebrovascular events: a prospective assessment with mri-initial results. Stroke $37: 818-823$

25. Sadat U, Teng Z, Young VE et al (2010) Association between biomechanical structural stresses of atherosclerotic carotid plaques and subsequent ischaemic cerebrovascular events-a longitudinal in vivo magnetic resonance imaging-based finite element study. Eur J Vasc Endovasc Surg 40:485-491

26. Teng Z, Brown AJ, Calvert PA et al (2014) Coronary plaque structural stress is associated with plaque composition and subtype and higher in acute coronary syndrome: the beacon $\mathrm{i}$ (biomechanical evaluation of atheromatous coronary arteries) study. Circ Cardiovasc Imaging 7:461-470

27. Teng Z, Degnan AJ, Sadat U et al (2011) Characterization of healing following atherosclerotic carotid plaque rupture in acutely symptomatic patients: an exploratory study using in vivo cardiovascular magnetic resonance. J Cardiovasc Magn Reson 13:64 